\title{
A Rare Case of Garengot's Hernia with Laparoscopic Approach in Colombia
}

\section{Martha Milena Alfonso-Gamba*, Maria Fernanda Mosquera-Gonzalez, Carlos Felipe Perdomo-Orozco, and Alberto Ricaurte-Aragón}

Department of General Surgery, Universidad del Rosario, Fundacion Santa Fe, Bogota, Colombia

*Corresponding author: Martha Milena Alfonso-Gamba, Department of General Surgery, Universidad del Rosario, Fundacion Santa Fe, Bogota, Colombia, Tel: 3142835376; E-mail: martha.alfonsog@gmail.com

Received: 17 Sep, 2019 | Accepted: 21 Nov, 2019 | Published: 27 Nov, 2019

Citation: Alfonso-Gamba MM, Mosquera-Gonzalez MF, Perdomo-Orozco CF, and Ricaurte-Aragón A (2019) A Rare Case of Garengot's Hernia with Laparoscopic Approach in Colombia. J Surg Open Access 5(6): dx.doi.org/10.16966/2470-0991.201

Copyright: (C) 2019 Alfonso-Gamba MM, et al. This is an open-access article distributed under the terms of the Creative Commons Attribution License, which permits unrestricted use, distribution, and reproduction in any medium, provided the original author and source are credited.

\section{Abstract}

Background: Garengeot's hernia is a rare presentation of a femoral hernia. Rarely, a Garengeot's hernia is managed laparoscopically.

Case report: We report a case of a 66 year-old male presented with an incarcerated groin hernia at the emergency department. He was taken in to laparoscopic hernia repair, with TAPP approach finding a right hernia femoral defect of $4 \mathrm{~cm}$ from which cecal appendix was reduced, in a suppurative phase.

Discussion: This is the fourth case reported in the literature. A Garengot's hernia can be managed laparoscopically. TAPP technique allows reducing the herniated appendix, repairing the hernia defect, determining the contamination of the abdominal cavity and the use of a mesh in the primary hernia repair.

\section{Introduction}

A femoral hernia is defined as a sac which projects through the femoral canal and presents clinically as a groin hernia below and laterals to the pubic tubercle or a finger breadth medial to the femoral artery below the inguinal ligament [1].

An inflamed appendix rarely can migrate in a hernia sac into the crural orifice and become incarcerated. The presence of the appendix in a femoral sac is known as a Garengeot's hernia [2] due to a French Surgeon called Rene Jacques Croissant de Garengeot who first described a case in 1731 [1-4]. The first appendectomy of a Garengeot's hernia was performed by Hevin in 1785 [2]. There is an incidence described in the literature from 0.08 to $1 \%[1,4]$.

A case of a Garengeot's hernia managed laparoscopically is presented at surgical emergency department in a fourth level hospital.

In a research done in pubmed, academic google, science direct and scielo databases there were 3 cases found.

\section{Case Report}

66 year old men arrived to the emergency department with history of 8 hours of right groin pain, sense of mass, nausea and vomit. He underwent right inguinal herniorrhaphy 30 years ago.

Clinical examination revealed good general conditions with normal vital signs and a non reductable painful mass in the right groin, without local inflammatory local changes such as erythema or edema and no signs of peritoneal irritation. Blood test with 16.000 leukocytes. There were no preoperative images done.
With diagnosis of an incarcerated groin hernia he was taken in to laparoscopic hernia repair finding a right femoral defect of $4 \mathrm{~cm}$ from which cecal appendix was reduced, in a suppurative phase (Figure 1). Without peritoneal fluid or additional inflammatory changes in the groin.

TAPP approach was done with an infraumbilical $12 \mathrm{~mm}$ trocar and other two trocars of $5 \mathrm{~mm}$ in right and left flanks. The appendix was easily reduced without perforating, mesoappendix was attached with ligasure and hemolock was placed at the base, appendix was extracted with endocath. We performed dissection of Bogro's and Retziu's space identifying Copper's ligament, iliopubic tract and deep inguinal ring.

The defect was corrected with a light polypropylene mesh of 15 $\times 15 \mathrm{~cm}$ fixed to the Cooper's ligament with 3 Protacks without making nearing and primary closure of the peritoneum with another 3 protacks avoiding contact of the mesh with the gut. Installation of drainage system was not considered.

No complications in the early postoperative period were presented. He was discharged 4 hours from hospital after surgery with analgesics. Pathology confirmed an acute suppurative appendicitis. The patient returns to normal activities on the seventh day after surgery.

\section{Discussion}

We present a rare case of Garengeot's hernia diagnosed and treated laparoscopically. This patient had an acute appendicitis and femoral hernia rarely seen, explored and treated at the same time. This case is the first reported in Colombia. 


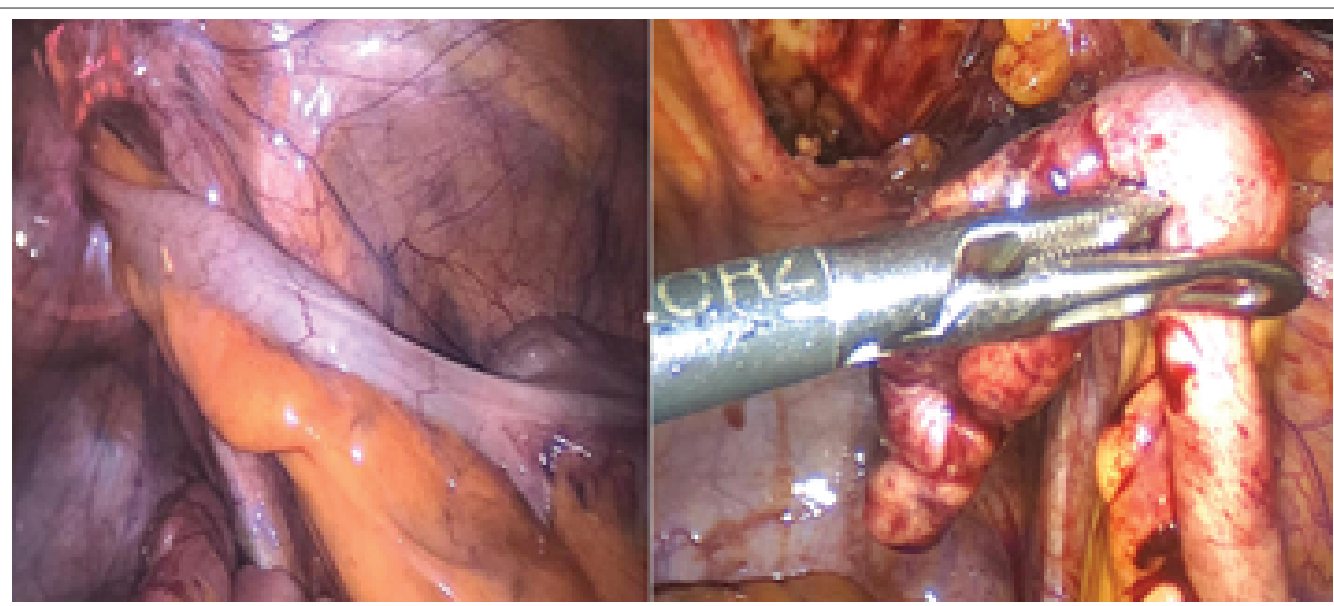

Figure 1: Left: Femoral defect with incarcerated appendix. Right: Appendix in a suppurative phase.

The treatment of Garengeot's hernia is appendectomy associated to primary hernia repair [1].

Since 1980's Transabdominal Preperitoneal (TAPP) and Total Extra Peritoneal (TEP) techniques have been broadly applied to the inguinal and femoral hernia repair, trying to diminish pain and hospitalization with many studies than prove it. These techniques have also been used in incarcerated hernias with good results. In Hospital Universitario Fundacion Santa Fe de Bogotá is the first line of treatment to the inguinal hernia repair.

Laparoscopic appendectomy can be performed in the same time of this procedure without any limitation. In 2003 and 2007, Comman A, et al. [4] documented this approach to an Amyand's and a Garengeot's hernia respectively. They were the first cases reported worldwide [1].

In 2016 Beysens M, et al. [1] and Díaz R, et al. [3] documented good results in the management of laparoscopic hernia associated to acute appendicitis.

In recent literature from 2015, there is a Systematic review about Garengeot hernia cases and its management results there were only 3 case reports repaired laparoscopically, the rest of them were open repairs [2]. Now days, the laparoscopic transabdominal approach is an option in addition to the open procedure.

As in this case, a patient with edematous appendix was a good candidate for the use of a mesh in the primary hernia repair. Comman A, et al. [4] argue that TAPP technique allow to determine the contamination of the abdominal cavity, and decide the applicability of a prosthesis $[1,4]$ instead of the TEP procedure.

Finally, installation of drainage of the abdominal cavity was not considered, formation of intraabdominal abscess is related with complicated forms of appendicitis, recent studies describe that placement of intra-abdominal drains in complicated acute appendicitis may not present benefits and may even lengthen hospital stay [5].

\section{Conclusion}

Laparoscopy with TAPP approach is an excellent alternative that diagnoses, evaluates the abdominal cavity and resolves the inflammatory condition (acute appendicitis) and femoral hernia at the same time.

\section{Conflict of Interest}

The authors report no conflict of interest and no funding was given for this purpose.

\section{References}

1. Beysens M, Haeck L, Vindevoghel K (2013) Laparoscopic appendectomy combined with TEP for de Garengeot hernia: case report. Acta Chir Belg 113: 468-470.

2. Al-Subaie S, Mustafa H, Al-Sharqawi N, Al-Haddad M, Othman F (2015) A case of de Garengeot hernia: the feasibility of laparoscopic transabdominal preperitoneal hernia repair. Int J Surg Case Rep 16: 73-76.

3. Díaz R, Rodríguez F, Bustos A, Alfaro J (2016) Cirugía laparoscópica de una hernia de Garengeot: presentación poco frecuente de una hernia crural. Rev Chil Cir 68: 446-448.

4. Comman A, Gaetzschmann P, Hanner T, Behrend M (2007) DeGarengeot hernia: transabdominal preperitoneal hernia repair and appendectomy. JSLS 11: 496-501.

5. Shlottmann F, Reino R, Sadava E, Campus A, Rotholltz NA (2016) Could an abdominal drainage be avoided in complicated acute appendicitis? Lessons learned after 1300 laparoscopic appendectomies. Int J Surg 36: 40-43. 